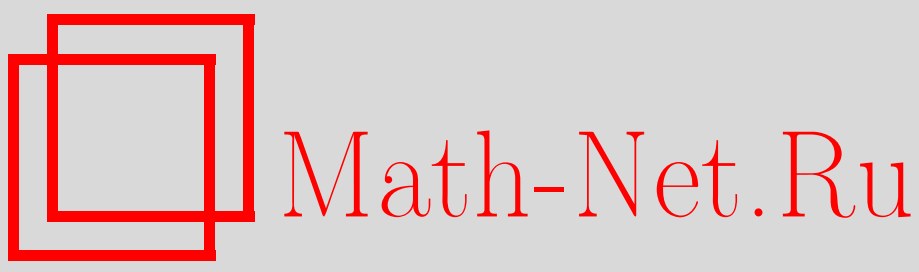

А. В. Ефимов, О краевых задачах с операторами М. Сайго для уравнения смешанного типа с дробной производной, Вестн. Сам. гос. техн. ун-та. Сер. Физ.-мат. науки, 2004, выпуск 26, 16-20

DOI: https://doi.org/10.14498/vsgtu171

Использование Общероссийского математического портала Math-Net.Ru подразумевает, что вы прочитали и согласны с пользовательским соглашением

http://www.mathnet.ru/rus/agreement

Параметры загрузки:

IP : 35.174 .16 .151

26 апреля 2023 г., 10:15:38

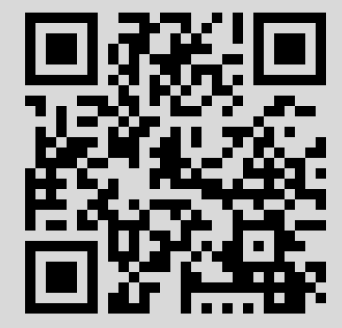




\section{О КРАЕВЫХ ЗАДАЧАХ С ОПЕРАТОРАМИ М.САЙГО ДЛЯ УРАВНЕНИЯ СМЕШАННОГО ТИПА С ДРОБНОЙ ПРОИЗВОДНОЙ}

Для уравнения смешанного типа с дробной производной поставлены и исследованы две нелокальные задачи в области, параболическая часть которой - верхняя полуплоскость. Отличительной особенностью данной работы является наличие обобщенных операторов в смысле М.Сайго в краевом условии. Доказана однозначная разрешимость обеих задач.

Введение. Рассмотрим уравнение

$$
0= \begin{cases}U_{x x}-D_{0+, y}^{\alpha} U, & y>0 ; \\ (-y)^{m} U_{x x}-U_{y y}=0, & y<0, m>-1, m \neq 0,\end{cases}
$$

которое имеет вырождение первого $(m>0)$ и второго рода $(-1<m<0)$. Здесь $D_{0+, y}^{\alpha}-$ частная дробная производная Римана-Лиувилля порядка $\alpha(0<\alpha<1)$ от функции $U(x, y)$ по второй переменной [1, с. 341]:

$$
\left(D_{0+, y}^{\alpha} U\right)(x, y)=\frac{d}{d y} \frac{1}{\Gamma(1-\alpha)} \int_{0}^{y} \frac{U(x, t)}{(y-t)^{1-\alpha}} d t, \quad(0<\alpha<1, y>0) .
$$

Обозначим через $J=(0,1)$ единичный интервал прямой $y=0 ;\left(I_{0+}^{\alpha} f\right)(x),\left(D_{0+}^{\alpha} f\right)(x)-$ операторы дробного интегродифференцирования в смысле Римана-Лиувилля; $\left(I_{0+}^{\alpha, \beta, \eta} f\right)(x)$ - обобщенный оператор дробного интегродифференцирования с гипергеометрической функцией Гаусса $F(a, b ; c ; z)$, введенный в [2] (см. также [1], с. 326-327) и имеющий при действительных $\alpha>0, \beta, \eta$ и $x>0$ вид

$$
\left(I_{0+}^{\alpha, \beta, \eta} f\right)(x)=\frac{x^{-\alpha-\beta}}{\Gamma(\alpha)} \int_{0}^{x}(x-t)^{\alpha-1} F\left(\alpha+\beta,-\eta ; \alpha ; 1-\frac{t}{x}\right) f(t) d t .
$$

Заметим, что

$$
\left(I_{0+}^{\alpha,-\alpha, \eta} f\right)(x)=\left(I_{0+}^{\alpha} f\right)(x),\left(I_{0+}^{-\alpha, \alpha, \eta} f\right)(x)=\left(D_{0+}^{\alpha} f\right)(x) .
$$

Вариант 1. Пусть $m>0, D=D^{+} \cup D^{-}$, где $D^{+}=\{(x, y):-\infty<x<\infty, y>0\}, D^{-}-$область, ограниченная характеристиками $A C: \xi=x-\frac{2}{m+2}(-y)^{\frac{m+2}{2}}=0, B C: \eta=x+\frac{2}{m+2}(-y)^{\frac{m+2}{2}}=1$ и отрезком [0,1] прямой $y=0, y<0$.

$\theta_{0}(x)$ - аффикс точки пересечения характеристики уравнения (1), выходящей из точки $(x, 0) \in J$, с характеристикой $A C$.

Для уравнения (1) изучим следующую нелокальную краевую задачу.

Задача 1. Найти решение $U(x, y)$ уравнения (1) в области $D$, удовлетворяющее краевым условиям:

$$
\begin{gathered}
\left.y^{1-\alpha} U\right|_{y=0}=0,-\infty<x \leq 0,1 \leq x<\infty, \\
A_{1} x^{1-2 \beta+b}\left(I_{0+}^{-\beta-c, b, c} t^{2 \beta-1} U\left[\theta_{0}(t)\right]\right)(x)+A_{2}\left(I_{0+}^{-c, 0,-1+2 \beta+c-b} U(t, 0)\right)(x)+ \\
+A_{3}\left(I_{0+}^{1-2 \beta-c, 2 \beta-1,-1+2 \beta+c-b} U_{y}(t, 0)\right)(x)=g(x), \quad x \in J,
\end{gathered}
$$

а также условиям сопряжения:

$$
\begin{gathered}
\lim _{y \rightarrow 0+} y^{1-\alpha} U(x, y)=\lim _{y \rightarrow 0-} U(x, y) \quad(x \in \bar{J}), \\
\lim _{y \rightarrow 0+} y^{1-\alpha}\left(y^{1-\alpha} U(x, y)\right)_{y}=\lim _{y \rightarrow 0-} U_{y}(x, y) \quad(x \in J) .
\end{gathered}
$$


Здесь $g(x) \in C^{1}(\bar{J}) \cap C^{2}(J)$ - заданная функция; $\beta=\frac{m}{2(m+2)}, 0<\beta<\frac{1}{2} ; A_{1}, A_{2}, A_{3}, c, b-$ действительные числа, причем $A_{1}$ и $A_{2}$ числа одинаковых знаков, $A_{1}$ и $A_{3}$ - противоположных знаков, а $c<0$.

Будем искать решение $U(x, y)$ поставленной задачи в классе дважды дифференцируемых функций в области $D$, таких, что

$$
\begin{gathered}
y^{1-\alpha} U(x, y) \in C\left(\overline{D^{+}}\right), U(x, y) \in C\left(\overline{D^{-}}\right), \\
y^{1-\alpha}\left(y^{1-\alpha} U\right)_{y} \in C\left(D^{+} \cup\{(x, y): 0<x<1, y=0\}\right), \\
U_{x x} \in C^{2}\left(D^{+} \cup D^{-}\right), U_{y y} \in C^{2}\left(\overline{D^{-}}\right) .
\end{gathered}
$$

Единственность решения задачи 1. Пусть существует решение исследуемой задачи. Введем обозначения:

$$
\begin{gathered}
\lim _{y \rightarrow 0+} y^{1-\alpha} U(x, y)=\tau_{1}(x), \lim _{y \rightarrow 0-} U(x, y)=\tau_{2}(x), \\
\lim _{y \rightarrow 0+} y^{1-\alpha}\left(y^{1-\alpha} U(x, y)\right)_{y}=v_{1}(x), \lim _{y \rightarrow 0-} U_{y}(x, y)=v_{2}(x) .
\end{gathered}
$$

Известно [3], что функциональное соотношение между $\tau_{1}(x)$ и $v_{1}(x)$, принесенное из параболической части $D^{+}$на линию $y=0$, имеет вид

$$
v_{1}(x)=\frac{1}{\Gamma(1+\alpha)} \tau_{1}^{\prime \prime}(x) .
$$

Найдем соотношение между $\tau_{2}(x)$ и $v_{2}(x)$, принесенное на линию $y=0$ из гиперболической части $D^{-}$области $D$. Используя формулу (19) из [4], имеем

$$
U\left[\theta_{0}(x)\right]=\gamma_{1} \Gamma(\beta)\left(I_{0+}^{\beta, 0, \beta-1} \tau_{2}\right)(x)-\gamma_{2} \Gamma(1-\beta)\left(I_{0+}^{1-\beta, 2 \beta-1, \beta-1} v_{2}\right)(x),
$$

где

$$
\gamma_{1}=\frac{\Gamma(2 \beta)}{\Gamma^{2}(\beta)}, \quad \gamma_{2}=\frac{1}{2}\left(\frac{4}{m+2}\right)^{2 \beta} \frac{\Gamma(1-2 \beta)}{\Gamma^{2}(1-\beta)} .
$$

Подставляя (12) в (4) и применяя ряд свойств обобщенных операторов дробного интегродифференцирования, получаем

$$
\tau_{2}(x)=K\left(I_{0+}^{1-2 \beta} v_{2}(t)\right)(x)+\Psi_{1}(x),
$$

где

$$
K=\frac{A_{1} \gamma_{2} \Gamma(1-\beta)-A_{3}}{A_{1} \gamma_{1} \Gamma(\beta)+A_{2}}, \Psi_{1}(x)=\frac{1}{A_{1} \gamma_{1} \Gamma(\beta)+A_{2}}\left(I_{0+}^{c, 0,-1+2 \beta-b} g(t)\right)(x) .
$$

Единственность решения задачи доказывается по схеме, предложенной в [4].

Сушествование решения задачи 1. Для доказательства существования решения задачи сведем ее к дифференциальному уравнению дробного порядка. Дифференцируя обе части (13) дважды по $x$, полагая $\tau_{1}(x)=\tau_{2}(x)=\tau(x), \nu_{1}(x)=v_{2}(x)=v(x)$ и учитывая (11), придем к дифференциальному уравнению дробного порядка $1+2 \beta$ :

$$
\left(D_{0+}^{1+2 \beta} v(t)\right)(x)-\frac{\Gamma(1+\alpha)}{K} v(x)=-\frac{\Psi_{1}^{\prime \prime}(x)}{K} .
$$

Известно (см. [1], прим. 42.1 и 42.2, с. 601-602), что общее решение дифференциального уравнения дробного порядка $\alpha>0$ вида

$$
\left(D_{0+}^{\alpha} y\right)(x)-\lambda y(x)=h(x) \quad(\alpha>0, n=-[-\alpha])
$$

задается формулой

$$
y(x)=\sum_{k=1}^{n} c_{k} x^{\alpha-k} E_{\alpha, 1+\alpha-k}\left(\lambda x^{\alpha}\right)+\int_{0}^{x}(x-t)^{\alpha-1} E_{\alpha, \alpha}\left[\lambda(x-t)^{\alpha}\right] h(t) d t .
$$

Здесь $c_{1}, \ldots, c_{n}$ - произвольные постоянные, а $E_{\alpha, 1+\alpha-k}\left(\lambda x^{\alpha}\right)$ и $E_{\alpha, 1+\alpha-k}\left[\lambda(x-t)^{\alpha}\right]-$ специальные случаи функции Миттаг-Леффлера $E_{\alpha, \beta}(z)$, определяемой равенством

$$
E_{\alpha, \beta}(z)=\sum_{m=0}^{\infty} \frac{z^{m}}{\Gamma(\alpha m+\beta)}(\alpha>0, \beta>0), E_{\alpha}(z) \equiv E_{\alpha, 1}(z)
$$


и являющейся целой функцией от $z$ (см. [5, 18.1], [1, с.33], [6, с.117]).

Уравнение (15) - уравнение вида (16) с $y(x)=v(x), \alpha=1+2 \beta, \lambda=\Gamma(1+\alpha) / K \quad$ и $h(x)=-\Psi_{1}^{\prime \prime}(x) / K$. Так как $0<\beta<\frac{1}{2}$, то $1<1+2 \beta<2$, и поэтому общее решение вида (17) для уравнения (15) с $K \neq 0$ дается формулой

$$
\begin{gathered}
v(x)=c_{1} x^{2 \beta} E_{1+2 \beta, 1+2 \beta}\left(\frac{\Gamma(1+\alpha)}{K} x^{1+2 \beta}\right)+c_{2} x^{2 \beta-1} E_{1+2 \beta, 2 \beta}\left(\frac{\Gamma(1+\alpha)}{K} x^{1+2 \beta}\right)- \\
-\frac{1}{K} \int_{0}^{x}(x-t)^{2 \beta} E_{1+2 \beta, 1+2 \beta}\left[\frac{\Gamma(1+\alpha)}{K}(x-t)^{1+2 \beta}\right] \Psi_{1}^{\prime \prime}(t) d t,
\end{gathered}
$$

где $c_{1}$ и $c_{2}$ - произвольные постоянные.

На основании работы [7] можно найти константы $c_{1}$ и $c_{2}$, а затем, используя (18), выписать явный вид для функции $\tau(x)$ :

$$
\begin{aligned}
& \tau(x)=c_{1} x E_{2 \beta+1,2}\left(\frac{\Gamma(1+\alpha)}{K} x^{2 \beta+1}\right)+c_{2} E_{2 \beta+1}\left(\frac{\Gamma(1+\alpha)}{K} x^{2 \beta+1}\right)- \\
& -\int_{0}^{x}(x-t) E_{2 \beta+1,2}\left[(x-t)^{2 \beta+1} \frac{\Gamma(1+\alpha)}{K}\right] \Psi_{1}^{\prime \prime}(t) d t+\Psi_{1}(x) .
\end{aligned}
$$

Вариант 2. Пусть $-1<m<0, D=D^{+} \cup D^{-}$, где $D^{+}$- область, описанная выше, а $D^{-}-$область, ограниченная характеристиками $A C: \xi=x-\frac{2}{2-m}(-y)^{\frac{2-m}{2}}=0$, $B C: \eta=x+\frac{2}{2-m}(-y)^{\frac{2-m}{2}}=1$ и отрезком [0,1] прямой $y=0, y<0$. Величина $\theta_{0}(x)-$ аффикс точки пересечения характеристики уравнения (1), выходящей из точки $(x, 0) \in J$, с характеристикой $A C$. Для уравнения (1) изучим следующую нелокальную краевую задачу.

Задача 2. Найти решение $U(x, y)$ уравнения (1) в области $D$, удовлетворяющее краевым условиям (3) и (4), а также условиям сопряжения (5) и (6). Здесь $g(x) \in C^{1}(\bar{J}) \cap C^{2}(J)-$ заданная функция; $\beta=\frac{m}{2(m+2)},-\frac{1}{2}<\beta<0 ; A_{1}, A_{2}, A_{3}, c, b$ - действительные числа, причем $A_{1}$ и $A_{2}$ числа одинаковых знаков, $A_{1}$ и $A_{3}$ - противоположных знаков, а $c<0$.

Будем искать решение $U(x, y)$ поставленной задачи в классе дважды дифференцируемых функций в области $D$, таких, что выполняются условия (7) и (8).

Единственность решения задачи 2. Пусть существует решение исследуемой задачи. Примем обозначения (9) и (10). Поскольку область $D^{+}$в задаче 2 та же, что и в задаче 1 , то первое функциональное соотношение между $\tau_{1}(x)$ и $v_{1}(x)$ будет иметь вид (11).

Найдем соотношение между $\tau_{2}(x)$ и $v_{2}(x)$, принесенное на линию $y=0$ из гиперболической части $D^{-}$области $D$. Известно [8], что обобщенное решение класса $R_{2}$ [9] уравнения (1) с начальными данными Коши

$$
\begin{gathered}
U(x,-0)=\tau_{2}(x)=\Gamma(1-2 \beta)\left(I_{0+}^{1-2 \beta} T\right)(x)=\int_{0}^{x} T(t)(x-t)^{-2 \beta} d t \quad(x \in \bar{J}), \\
\lim _{y \rightarrow 0-} U_{y}(x, y)=[2(1-2 \beta)]^{-2 \beta} . \lim _{(\eta-\xi) \rightarrow 0-}(\eta-\xi)^{2 \beta}\left(U_{\xi}-U_{\eta}\right)=v_{2}(x) \quad(x \in J)
\end{gathered}
$$

можно представить в виде

где

$$
\begin{gathered}
U(\xi, \eta)=\int_{0}^{\xi} T(t)(\eta-t)^{-\beta}(\xi-t)^{-\beta} d t+ \\
+\frac{1}{2 \cos \pi \beta} \int_{\xi}^{\eta} T(t)(\eta-t)^{-\beta}(t-\xi)^{-\beta} d t-k_{0} \int_{\xi}^{\eta} v_{2}(t)(\eta-t)^{-\beta}(t-\xi)^{-\beta} d t \\
k_{0}=\frac{\Gamma(2-2 \beta)}{\Gamma^{2}(1-\beta)}[2(1-2 \beta)]^{2 \beta-1}
\end{gathered}
$$


$T(x)$ и $v_{2}(x)$ непрерывны в интервале $(0,1)$ и интегрируемы на отрезке $[0,1]$.

Из формулы (20) имеем

$$
U\left[\theta_{0}(x)\right]=I_{0+}^{1-\beta, 2 \beta-1, \beta-1}\left[\frac{\Gamma(1-\beta)}{2 \cos \pi \beta} T(x)-k_{0} v_{2}(x) \Gamma(1-\beta)\right] .
$$

Подставляя (21) в (4), применяя ряд свойств обобщенных операторов дробного интегродифференцирования, получаем

$$
\mathrm{v}_{2}(x)=K_{1} T(x)+C(x)
$$

где

$$
K_{1}=\frac{A_{1} \frac{\Gamma(1-\beta)}{2 \cos \pi \beta}+A_{2} \Gamma(1-2 \beta)}{A_{1} k_{0} \Gamma(1-\beta)-A_{3}}, K_{2}=\frac{1}{A_{1} k_{0} \Gamma(1-\beta)-A_{3}}, C(x)=K_{2}\left(I_{0+}^{-1+2 \beta+c, 1-2 \beta,-b} g(t)\right)(x) .
$$

Или, воспользовавшись (19), имеем

$$
\mathrm{v}_{2}(x)=K_{1}\left(D_{0+}^{1-2 \beta} \tau_{2}(t)\right)(x)+C(x) .
$$

При $g(x) \equiv 0$ из (23) получим

$$
v_{2}(x)=K_{1}\left(D_{0+}^{1-2 \beta} \tau_{2}(t)\right)(x)
$$

Единственность решения задачи вытекает из аналога принципа экстремума А.В. Бицадзе [10].

Пусть $\underset{D^{+}}{\max } U(x, y)=\tau_{2}\left(x_{0}\right)>0$. Тогда, в соответствии с принципом экстремума для операторов дробного дифференцирования [11], из (24) и условия $K_{1}>0$ заключаем, что $v_{2}\left(x_{0}\right)>0$. Поскольку $\tau^{\prime \prime}\left(x_{0}\right)=0$, то из (11) заключаем, что $v_{1}\left(x_{0}\right)=0$.

Итак, $v_{2}\left(x_{0}\right)>0$, а $v_{1}\left(x_{0}\right)=0$, что противоречит условию сопряжения (6), откуда и следует единственность решения задачи 2 .

Существование решения задачи 2. Для доказательства существования решения исходной задачи сведем ее к интегральному уравнению дробного порядка. Из (19) с помощью второго выражения из (2) имеем

$$
T(x)=\frac{1}{\Gamma(1-2 \beta)}\left(I_{0+}^{2 \beta-1,1-2 \beta, \eta} \tau(t)\right)(x) .
$$

Выражая $\tau_{1}(x)$ из соотношения (11), используя первое выражение из (2), получаем

$$
\tau_{1}(x)=\Gamma(1+\alpha)\left(I_{0+}^{2,-2, \eta+2 \beta-1} v_{1}(t)\right)(x) .
$$

Учитывая (25) и полагая $\tau_{1}(x)=\tau_{2}(x)=\tau(x)$ и $\mathrm{v}_{1}(x)=\mathrm{v}_{2}(x)=v(x)$, будем иметь

$$
\begin{gathered}
T(x)=\frac{\Gamma(1+\alpha)}{\Gamma(1-2 \beta)} I_{0+}^{2 \beta-1,1-2 \beta, \eta}\left(I_{0+}^{2,-2, \eta+2 \beta-1} v(t)\right)(x)=\frac{\Gamma(1+\alpha)}{\Gamma(1-2 \beta)}\left(I_{0+}^{1+2 \beta,-1-2 \beta, \eta} v(t)\right)(x)= \\
=\frac{\Gamma(1+\alpha)}{\Gamma(1-2 \beta)}\left(I_{0+}^{1+2 \beta} v(t)\right)(x) .
\end{gathered}
$$

Подставляя полученное значение $T(x)$ в (22), приходим к интегральному уравнению дробного порядка

$$
v(x)=B_{1}\left(I_{0+}^{1+2 \beta} v(t)\right)(x)+C(x),
$$

где

$$
B_{1}=K_{1} \frac{\Gamma(1+\alpha)}{\Gamma(1-2 \beta)}
$$

Уравнение (26) можно переписать в виде:

$$
v(x)=C(x)+\frac{B_{1}}{\Gamma(1+2 \beta)} \int_{0}^{x} v(t)(x-t)^{2 \beta} d t .
$$

Далее воспользуемся следующей теоремой (см. [6, с. 123]).

Теорема. Пусть функция $f(x) \in L[a, b]$. Тогда интегральное уравнение 


$$
u(x)=f(x)+\frac{\lambda}{\Gamma(1 / \rho)} \int_{a}^{x}(x-t)^{1 / \rho-1} u(t) d t,
$$

где $\rho>0, \lambda$ - произвольный комплексный параметр, имеет единственное решение

$$
u(x)=f(x)+\lambda \int_{a}^{x}(x-t)^{1 / \rho-1} E_{\rho}\left[\lambda(x-t)^{1 / \rho} ; 1 / \rho\right] f(t) d t,
$$

принадлежащие $L[a, b]$.

Здесь

$$
E_{\rho}(z ; \mu)=\sum_{k=0}^{\infty} \frac{z^{k}}{\Gamma(\mu+k / \rho)} \equiv E_{1 / \rho, \mu}(z)
$$

- функция типа Миттаг-Леффлера, которая является целой функцией (комплексной) переменной $z=x+i y$ порядка $\rho>0$ (см. [6, с. 117]). При $\mu=1$ функция (28) совпадает с функцией Миттаг-Леффлера $E_{1 / \rho}(z) \equiv E_{\rho}(z ; 1)$. Поэтому решение уравнения (27) будет иметь вид

$$
v(x)=C(x)+A_{1} \int_{0}^{x}(x-t)^{2 \beta} E_{\frac{1}{1+2 \beta}}\left[A_{1}(x-t)^{1+2 \beta} ; 1+2 \beta\right] C(t) d t .
$$

Используя явный вид функций $\tau(x)$ и $v(x)$, можно получить решения задач 1 и 2 в каждой из областей $D^{+}$и $D^{-}$, а, значит, и решения задач в заданном классе функций в области $D$, удовлетворяющие краевым условиям (3), (4) и условиям склеивания (5), (6).

\section{БИБЛИОГРАФИЧЕСКИЙ СПИСОК}

1. Самко С.Г., Килбас А.А., Маричев О.И. Интегралы и производные дробного порядка и некоторые их приложения. Минск: Наука и техника, 1987. 688 с.

2. Saigo M. A remark on integral operators involving the Gauss hypergeometric functions. // Math. Rep. Kyushu Univ. 1978. Vol. 11. №. 2. P. 135-143.

3. Геккиева C.X. Об одном аналоге задачи Трикоми для уравнения смешанного типа с дробной производной. // Докл. АМАН. 2001. Т. 5. №. 2. С. 18-22.

4. Килбас А.А. Репин О.А. Задача со смещением для параболо-гиперболического уравнения. // Дифференц. уравнения. 1998. Т. 34. №. 6. С. 799-805.

5. Бейтмен Г., Эрдейи А. Высшие трансцендентные функции. Т. 3. М.: Наука, 1967. 234 с.

6. Джрбашян М.M. Интегральные преобразования и представления функции в комплексной области. М.: Наука, 1966. $672 \mathrm{c}$

7. Килбас А.А., Репин О.А. Аналог задачи Бицадзе-Самарского для уравнения смешанного типа с дробной производной. // Дифференц. уравнения. 2003. Т. 39. №. 5. С. 638-644.

8. Смирнов М.М. Уравнения смешанного типа. М.: Высшая школа, 1985.304 с.

9. Кароль И.Л. Об одной краевой задаче для уравнения смешанного эллиптико-гиперболического типа. // Докл. АН СССР. 1953. Т. 88. № 2. С. 197-200.

10. Бицадзе A.B. Некоторые классы уравнений в частных производных. М.: Наука, 1981. 448 с.

11. Нахушев А.М. Уравнения математической биологии. М.: Высшая школа, 1995. 301 с. 